\title{
Sensibilité variétale d'un blé d'hiver à la concurrence d'une avoine adventice : influence sur le seuil biologique de nuisibilité
}

\author{
G. Mondragon ${ }^{1 *}$, J.P. Caussanel ${ }^{1}$, C. Aujas ${ }^{1}$, A. Carteron ${ }^{2}$ et J. Schiex ${ }^{3}$ \\ 1 INRA, laboratoire de malherbologie, B.V. 1540, 21034 Dijon Cedex; \\ 2 INRA, station d'amélioration des plantes, B.V. 1540, 21034 Dijon Cedex; \\ 3 GEVES, B.V. 1540, 21034 Dijon Cedex, France
}

(reçu le 17 janvier 1989, accepté le 30 mai 1989)

Résumé - Les relations de concurrence bispécifique entre une adventice dominante, l'avoine, et une plante cultivée, le blé, ont été étudiées en considérant certains aspects démographiques, génétiques et fonctionnels.

En plein champ, un seuil biologique moyen de nuisibilité d'une avoine d'hiver semée dans une culture de blé d'hiver en même temps que le blé a été déterminé. II se situe entre 1 et 4 plantes d'avoine par $\mathrm{m}^{2}$. L'aptitude à la concurrence de la plante cultivée est liée à des différences génétiques : l'un des 4 cultivars de blé a légèrement mieux résisté à la concurrence de l'avoine d'hiver. L'augmentation de la densité d'avoine d'hiver a principalement entraîné une réduction du nombre d'épis de blé par plante et du poids de 1000 grains. Aucun effet sur le rendement et les composantes du rendement du blé d'hiver n'a été constaté en semant une avoine de printemps au stade trois feuilles du blé et à des densités comprises entre 0 et 15 plantes d'avoine par $\mathrm{m}^{2}$.

L'aptitude à la concurrence de l'avoine peut s'expliquer en mesurant, à des périodes déterminées, certains paramètres de croissance et de développement (aspects fonctionnels) des 2 espèces en concurrence. Le rythme d'apparition des feuilles du brin maître et des talles secondaires du blé n'est pas modifié par la concurrence de l'avoine.

concurrence interspécifique - céréale - graminée adventice - variété ou cultivar

Summary - Response of different wheat cultivars to oat competition : effect on the biological threshold. The relations of competition between a dominant weed species (oat) and a crop plant (wheat) were studied, taking into account some demographic, genetic and functional aspects.

In field conditions, an average biological threshold was determined for a winter oat sown in a winter wheat crop; both species were sown at the same time. This threshold lies between 1 and 4 oat plants per $\mathrm{m}^{2}$. Wheat competitive ability was related to genetic differences; one of the 4 wheat cultivars offered a slightly greater aggressivity to winter oat competition. The increase in winter oat density mainly caused a reduction in the number of wheat ears per plant and in the weight of 1000 grains. No effect on wheat yield or yield components was shown by sowing a spring oat at the three-leaf stage of wheat at a range density of 0 to 15 oats per $\mathrm{m}^{2}$.

Oat competitive ability can be explained by measuring some growth and development parameters at well-defined growth stages (functional aspects) of both species in competition. The rhythm of leaf emergence on the wheat main shoot and wheat tiller development were not altered by oat competition.

interspecific competition - cereal - grass weed - variety or cultivar

\section{INTRODUCTION}

Diverses espèces de folles avoines ont été décrites en France et deux d'entre elles sont considérées depuis longtemps comme nuisibles pour l'agriculture (Barralis, 1961). Ces 2 espèces, Avena fatua L. et Avena sterilis L. ssp ludoviciana (Durieu) Nyman, présentent une fréquence et une abondance élevées dans cer- taines régions de culture du blé d'hiver (Barralis, 1977).

Plusieurs facteurs climatiques et édaphiques agissent sur les caractéristiques de croissance et de développement de ces espèces (Chancellor, 1976). Par ailleurs, les sévères réductions de rendement de la céréale dues à la présence de folles avoines varient avec les facteurs agrotechniques, qui peuvent avantager ou désavantager

\footnotetext{
*Adresse actuelle : Dpto. de campo experimental, Universidad autonoma chapingo, Chapingo, Mexico, C.P. 56230 (Mexique)
} 
l'espèce cultivée (Chancellor \& Peters, 1976). Les interactions biologiques entre une espèce adventice et une espèce cultivée présentent aussi des aspects génétiques (Caussanel, 1989), qui impliquent autant l'utilisation de variétés ou de cultivars pour l'espèce cultivée (Glauninger \& Holzner, 1982) que la variabilité inter et intrapopulation des populations adventices (Harlan, 1982).

Des pertes de rendement ont été mesurées expérimentalement sur des cultivars de blé soumis à la concurrence de la folle avoine (Avena fatua L.), mais les auteurs s'interrogent sur l'interprétation biologique des différences constatées (Holmes \& Pfeiffer, 1962). La diversité des blés tendres cultivés en France (Branlard \& Chevalet, 1984) conduit à comparer leur compétitivité vis-àvis des mauvaises herbes, d'autant plus que chaque variété possède son propre rythme d'apparition des feuilles (Kirby et al., 1985), dans la logique d'un fonctionnement intégré du semis à l'épiaison (Malvoisin, 1984). En Bourgogne, plusieurs cultivars de blé d'hiver à haute potentialité de rendement sont recommandés, mais leur réponse à la concurrence de graminées adventices comme la folle avoine est encore peu connue. Le dispositif expérimental agronomique utilisé dans l'étude de la concurrence entre une avoine de printemps et un blé de printemps (Caussanel \& Kafiz, 1986) a permis d'analyser le seuil biologique de nuisibilité d'une avoine cultivée considérée comme adventice, dans une culture de blé d'hiver, sous ses aspects démographiques et fonctionnels. Dans les essais décrits ci-après, l'utilisation de plusieurs cultivars de blé d'hiver avait pour but d'inclure les aspects génétiques en étudiant l'effet variétal de la plante cultivée dans les phénomènes de concurrence entre les 2 graminées. II s'agissait principalement de relier la diminution de rendement du blé d'hiver, selon le cultivar utilisé, à la densité d'infestation d'avoine.

\section{MATÉRIEL ET MÉTHODES}

Deux expériences ont été menées au domaine expérimental d'Epoisses (INRA, Dijon) en Côte-d'Or, l'une en 1985-1986, l'autre en 1986-1987.

\section{Matériel végétal}

\section{Blé}

Quatre cultivars de blé d'hiver, identiques d'une année à l'autre, sont utilisés. Ils sont choisis en fonction de leur bonne productivité dans la région Bourgogne et de leurs différences respectives en capacité de tallageépis et en hauteur de paille:
- Talent : capacité de tallage forte et hauteur de paille courte

- Fidel : capacité de tallage assez forte et hauteur de paille moyenne

- Capitole et Castan (blé alternatif) : capacité de tallage moyenne et hauteur de paille assez courte.

\section{Avoine}

Une avoine d'hiver (cv. Fringante) est employé la première année et une avoine de printemps (cv. Selma) la deuxième année.

\section{Conditions de culture}

Les essais sont implantés sur un sol limono-argileux après une culture de pois protéagineux de printemps l'année précédente et d'orge de printemps l'année antérieure. La parcelle d'essai est fertilisée avec 110 unités de $\mathrm{P}_{2} \mathrm{O}_{5}$ et $\mathrm{K}_{2} \mathrm{O}$ à l'automne et avec 150 unités d'azote fournies en 2 apports équivalents au printemps: au tallage et à la montaison du blé. Les semis du blé d'hiver et de l'avoine d'hiver sont effectués simultanément (29 octobre 1985 pour le premier essai et 24 octobre 1986 pour le deuxième essai), ce qui permet d'obtenir des levées concomitantes, dans de petites parcelles de $7 \mathrm{~m}$ de longueur et de $1,25 \mathrm{~m}$ de largeur, dont $5,4 \mathrm{~m}^{2}$ sont récoltables. Chacune des parcelles comporte 6 lignes de blé et 7 lignes d'avoine semées dans l'inter-rang du blé, qui est de $15 \mathrm{~cm}$. Le blé est semé à la densité de 250 semences par $\mathrm{m}^{2}$ et l'avoine aux densités prévues dans le dispositif expérimental agronomique. Le semis d'avoine de printemps est réalisé dans l'inter-rang du blé ( 9 mars 1987) à des densités au moins 2 fois supérieures aux densités prévues. Un éclaircissage des plantules surnuméraires est pratiqué peu après la levée ( 6 avril 1987).

Les autres adventices sont supprimées par un traitement herbicide différent chaque année :

- 1985-1986 : dichlorprop (1 $225 \mathrm{~g} / \mathrm{ha}$ ) + MCPA (350 g/ha) + mecoprop (525 g/ha) appliqué au stade mi-tallage du blé le 6 mai 1986;

- 1986-1987 : trifluraline (960 g/ha) + linuron ( $480 \mathrm{~g} / \mathrm{ha}$ ) appliqué en prélevée le 27 octobre 1986.

Le dispositif expérimental est celui du split-plot à 2 facteurs et comporte 5 répétitions. Dans chaque expérience, les 2 facteurs étudiés sont les suivants :

\section{Facteur principal ( $V i$, à 4 niveaux)}

4 cultivars de blé d'hiver :

$-\mathrm{V} 1$ = Fidel;

- V2 = Capitole;

- V3 = Castan;

- V4 = Talent;

\section{Facteur subsidiaire $(D j)$}

\section{A 3 niveaux en 1985-1986 (3 densités de concurrence de l'avoine d'hiver)}

- DO = aucune semence par $\mathrm{m}^{2}$;

-D1 = 10 semences par $\mathrm{m}^{2}$;

-D2 $=20$ semences par $\mathrm{m}^{2}$ 
A 7 niveaux en 1986-1987 (7 densités de concurrence de l'avoine de printemps)

- DO = aucune plante par $\mathrm{m}^{2}$;

- D1 = 2,5 plantes par $\mathrm{m}^{2}$;

- D2 = 5 plantes par $\mathrm{m}^{2}$;

- D3 $=7,5$ plantes par $\mathrm{m}^{2}$;

- D4 = 10 plantes par $\mathrm{m}^{2}$;

- D5 = 12,5 plantes par $\mathrm{m}^{2}$;

- D6 = 15 plantes par $\mathrm{m}^{2}$.

\section{Mesures et comptages}

Chacune des parcelles élémentaires $V i$ Dj en 1985 1986 et Vi D0, Vi D2, Vi D4 et ViD6 en 1986 est répétée 2 fois dans le dispositif, de façon à effectuer des prélèvements de plantes sur des parcelles spécialement réservées à cet effet («parcelle de prélèvement»). Dans ces parcelles de prélèvement, l'évolution du développement du blé est suivie en fonction du temps (sur la variété Talent en 1985-1986 et sur toutes les variétés en 1986-1987). Les stades phénologiques sont déterminés selon une méthode décrite antérieurement (Malvoisin, 1984). Sur les parcelles destinées à la récolte ("parcelle de récolte»), les levées de blé sont comptées au stade 3 feuilles à 1 talle du blé $(2$ avril 1986 : stade 1 talle, 4 mars 1987 : stade 3 feuilles). De plus, des comptages d'avoine sont effectués au stade début tallage du blé ( 2 avril 1986 et 14 avril 1987). Enfin, des comptages d'épis et de panicules ont lieu au moment de la maturation (15 au 31 juillet 1986 et 1987). Ces comptages sont réalisés sur 2 placettes fixes de $0,45 \mathrm{~m}^{2}$ disposées à chaque extrémité de la parcelle. Les composantes du rendement sont mesurées sur ces placettes fixes quelques jours avant la récolte. Puis l'avoine est enlevée manuellement sur toutes les parcelles avant la récolte du blé (30 juillet 1986 et 4 août 1987), faite mécaniquement avec une micromoissonneuse-batteuse.

Sur les parcelles de récolte comme sur les placettes de chacune d'entre elles, les rendements sont mesurés ainsi que l'humidité des grains, afin d'exprimer chaque rendement à $15 \%$ d'humidité. Les pourcentages de pertes de rendement (\%) dus à chaque densité d'avoine sont calculés ainsi :

$$
(\%)=\frac{Y_{i 0}-Y_{i j}}{Y_{i 0}} \times 100
$$

où Yio représente le rendement du témoin sans avoine à la densité $\mathrm{DO}$ (parcelles $V i \mathrm{DO}$ ) et $Y i j$ le rendement de la parcelle correspondante à la densité $\mathrm{D} j$ présente à la récolte.

\section{Traitement des résultats}

Les résultats sont traités statistiquement par la méthode d'analyse de variance d'un dispositif split-plot à 2 facteurs (Snedecor \& Cochran, 1971). Les effets de chacun des 2 facteurs et les interactions significatives sont recherchés. Lorsque l'interaction entre la densité d'avoine et le cultivar de blé est significative, la partition de la somme des carrés correspondante est effectuée (Little, 1981) et des équations de régression calculées à l'aide de programmes statistiques (logiciel SAS).

\section{RÉSULTATS ET DISCUSSION}

\section{Evolution de la densité d'avoine au cours du temps}

En 1986, les comptages de levées d'avoine effectués au stade 1 talle de blé ont montré que les densités de peuplement sont réduites à 50 $70 \%$ par rapport aux densités de semis. A partir de cette date, les densités de peuplement de l'avoine sont restées approximativement constantes jusqu'à la récolte (Fig. 1a). Le nombre de panicules d'avoine par $\mathrm{m}^{2}$ varie peu entre les 4 cultivars : 23 à 27 pour la densité D1 et 33 à 42 pour la densité D2 (Fig. 1b). Tous cultivars confondus, la densité moyenne de l'avoine au début du tallage est de 5,1 plantes par $\mathrm{m}^{2}$ pour la densité $\mathrm{D} 1$ et de 8,2 plantes par $\mathrm{m}^{2}$ pour la densité D2 (Tableau la). Quel que soit le cultivar, le nombre de panicules par plante est toujours plus faible à la densité D2 (4,4 panicules par plante en moyenne) qu'à la densité D1 $(5,2$ panicules par plante en moyenne).

En 1987, le nombre de pieds d'avoine à la maturation du blé est réduit de $20-25 \%$ en moyenne par rapport à la densité de peuplement obtenue par éclaircissage au stade 3 feuilles du blé, excepté pour le cultivar Castan (Tableau lb et Fig. 1C). Comme en 1986, les densités moyennes de panicules par $\mathrm{m}^{2}$ à maturation, sont différentes pour chaque densité de population d'avoine présente au stade 3 feuilles du blé. En revanche, le nombre de panicules par plante est plus faible qu'en 1986 et ne diminue pas avec la densité. L'effet de concurrence intraspécifique
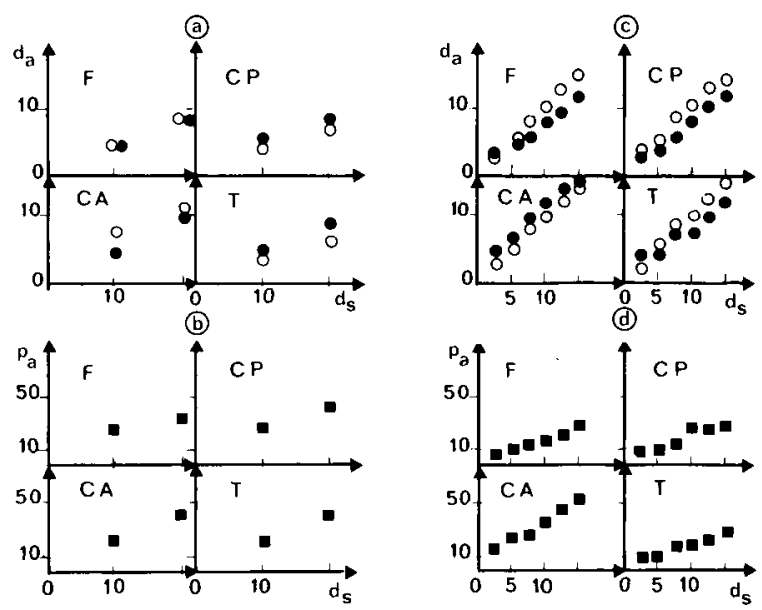

Fig. 1. Relation entre la densité de peuplement et la densité de semis d'avoine d'hiver dans une culture de blé d'hiver pour différents cultivars de blé.

a, b : essai 1985-1986; c, d : essai 1986 - 1987; ds : densité de semis; $d_{a}$ : Densité estimée (en nombre de plantes d'avoine par $\mathrm{m}^{2}$ )

$O$ : au stade 3 feuilles (1987), ou 1 talle (1986) du blé; $\bullet$ : à maturation

$\mathrm{p}_{\mathrm{a}}$ : Densité estimée (en nombre de panicules d'avoine par $\mathrm{m}^{2}: \mathbf{E}$ ); F : Fidel; CP : Capitole; CS : Castan; $\mathrm{T}$ : Talent. 
Tableau I. Densités moyennes d'avoine dans les parcelles de récolte.

\begin{tabular}{|c|c|c|c|c|}
\hline $\begin{array}{l}\text { Densité } \\
\text { d'avoine }\end{array}$ & \multicolumn{2}{|c|}{$\begin{array}{c}\text { Nombre de pieds d'avoine } \\
\text { par } m^{2}\end{array}$} & \multicolumn{2}{|c|}{$\begin{array}{c}\text { Nombre de panicules d'avoine } \\
\text { à maturation }\end{array}$} \\
\hline $\begin{array}{l}\text { par } m^{2} \\
\text { (1) }\end{array}$ & $\begin{array}{l}\text { à la reprise } \\
\text { de végétation (2) }\end{array}$ & à maturation & par m² & par plante \\
\hline
\end{tabular}

a) Essai $1985-1986$

$\begin{array}{rllll}0 & 0 & 0 & 0 & 0 \\ 10 & 5,1 & 4,6 & 24,0 & 5,2 \\ 20 & 8,2 & 8,9 & 39,2 & 4,4\end{array}$

b) Essai 1986 - 1987

\begin{tabular}{|c|c|c|c|c|}
\hline 0 & 0 & 0 & 0 & 0 \\
\hline 2,5 & 2,7 & 3,4 & 10,3 & 3,0 \\
\hline 5 & 5,0 & 4,8 & 14,0 & 2,8 \\
\hline 7,5 & 7,9 & 7,0 & 19,0 & 2,6 \\
\hline 10 & 10,0 & 8,8 & 25,2 & 2,9 \\
\hline 12,5 & 12,5 & 10,6 & 28,9 & 2,7 \\
\hline 15 & 14,5 & 12,9 & 34,4 & 2,7 \\
\hline
\end{tabular}

(1) Essai 1985-1986: densité de semis

Essai 1986-1987 : densité de peuplement après éclaircissage

(2) Essai 1985-1986 : densité d'avoine d'hiver au stade 1 talle du blé

Essai 1986-1987 : densité d'avoine de printemps au stade 3 feuilles du blé

est moins marqué pour l'avoine de printemps que pour l'avoine d'hiver, aux niveaux de densité choisis. A densité égale, le nombre de pieds et de panicules d'avoine par $\mathrm{m}^{2}$ diffère peu entre Fidel, Capitole et Talent, alors que l'avoine produit plus de pieds et environ 2 fois plus de panicules par $\mathrm{m}^{2}$ en présence de Castan (Fig. 1c et 1d). Le meilleur développement de l'avoine dans ce dernier cas pourrait s'expliquer par la plus grande sensibilité au froid du cultivar Castan : les plantules survivantes à la reprise de végétation ont plus souffert de la concurrence de l'avoine que celles des autres cultivars (Tableau II). Dans d'autres conditions environnementales, il a été prouvé que des cultivars de blé, exigeants quant à leurs besoins en vernalisation, manifestent une tendance à être défavorisés dans leur concurrence avec Lolium rigidum Gaud (Flood \& Halloran, 1984).

\section{Influence de la densité d'avoine sur le rende- ment du blé}

Lorsque le blé n'est pas soumis à la concurrence de l'avoine, les rendements parcellaires varient, selon le cultivar utilisé, de $65,5 \mathrm{q} / \mathrm{ha}$ à $75,5 \mathrm{q} / \mathrm{ha}$ en 1986 et de 67,5 q/ha à 75,9 q/ha en 1987 (Tableau III). En situation de concurrence avec l'avoine, il existe, chaque année, un effet haute-

Tableau II. Densités moyennes de blé par cultivar, estimées à différents stades dans les parcelles de récolte.

Essai 1985-1986

Cultivar

Nombre de plantes

de blé par $m^{2}$

au stade 1 talle

2 avril 1986

Essai 1986-1987

Nombres de plantes de blé par $m^{2}$

au stade 1 feuille

du blé

19 novembre 1986 au stade 3 feuilles du blé

4 mars 1987

$\begin{array}{lllr}\text { Fidel } & 178,5 & 177,8 & 180,6 \\ \text { Capitole } & 188,2 & 174,5 & 180,9 \\ \text { Castan } & 217,9 & 157,3 & 127,5 \\ \text { Talent } & 201,3 & 195,3 & 178,7\end{array}$


Tableau III. Effet de l'année et du cultivar de blé sur le rendement du blé d'hiver dans les parcelles témoins sans avoine.

\begin{tabular}{lcc}
\hline & \multicolumn{2}{c}{ Rendement parcellaire (en q/ha) } \\
Cultivar & $1985-1986$ & $1986-1987$ \\
& & \\
\hline & & 72,9 \\
Fidel & 75,3 & 71,2 \\
Capitole & 65,5 & 67,5 \\
Castan & 72,8 & 75,9 \\
Talent & 75,5 & \\
\hline
\end{tabular}

ment significatif $(P<0,01)$ du cultivar de blé (Tableau IV). Le rendement du cultivar Capitole est inférieur chaque année à celui de Talent, cultivar considéré comme témoin en raison de son utilisation fréquente en Bourgogne.

L'effet de la densité d'avoine sur le rendement ne se manifeste qu'en 1986 : la perte moyenne de rendement s'élève déjà à $6,1 \%$ à la densité de 5,1 plantes d'avoine d'hiver par $\mathrm{m}^{2}$. En 1987, les pertes moyennes de rendement atteignent seulement $1,4 \%$ et $3,2 \%$ aux densités moyennes respectives d'avoine de printemps de 5,0 à 10,0 plantes par $\mathrm{m}^{2}$ au stade 3 feuilles de blé. L'absence d'effet significatif $(P>0,05)$ de la densité d'avoine de printemps sur le rendement du blé d'hiver en 1987 est à mettre en relation avec

Tableau IV. Influence de la densité d'avoine sur le rendement de différents cultivars de blé d'hiver.

\begin{tabular}{|c|c|c|c|c|c|}
\hline \multirow[b]{2}{*}{ Traitement } & \multicolumn{2}{|c|}{ Essai 1985-1986 } & \multirow[b]{2}{*}{ Traitement } & \multicolumn{2}{|c|}{ Essai 1986-1987 } \\
\hline & $\begin{array}{l}\text { Rendement } \\
\text { (en q/ha) }\end{array}$ & $\begin{array}{c}\text { Augmentation ou } \\
\text { perte de rendement } \\
\text { par rapport au témoin } \\
(\%)\end{array}$ & & $\begin{array}{c}\text { Rendement } \\
\text { (en q/ha) }\end{array}$ & $\begin{array}{c}\text { Augmentation ou } \\
\text { perte de rendement } \\
\text { par rapport au témoin } \\
(\%)\end{array}$ \\
\hline Cultivar & & & Cultivar & & \\
\hline Fidel & 71,9 & $+2,9$ & Fidel & 73,0 & $-2,8$ \\
\hline Capitole & 62,5 & $-10,6$ & Capitole & 68,7 & $-8,5$ \\
\hline Castan & 69,1 & $-1,1$ & Castan & 65,5 & $-12,8$ \\
\hline Talent (témoin) & 69,9 & - & Talent (témoin) & 75,1 & - \\
\hline $\begin{array}{l}\text { Densité } \\
\text { de population } \\
\text { d'avoine d'hiver } \\
\text { au stade de } \\
1 \text { talle du blé }\end{array}$ & & & $\begin{array}{c}\text { Densité } \\
\text { de population } \\
\text { d'avoine de printemps } \\
\text { au stade } 3 \text { feuilles } \\
\text { du blé }\end{array}$ & & \\
\hline 0 & 72,3 & - & $\begin{array}{l}0 \\
2,7\end{array}$ & $\begin{array}{l}71,9 \\
70,6\end{array}$ & $-\overline{1,8}$ \\
\hline 5,1 & 67,9 & $-6,1$ & $\begin{array}{l}5,0 \\
7,9\end{array}$ & $\begin{array}{l}70,9 \\
69,7\end{array}$ & $\begin{array}{l}-1,4 \\
-3,1\end{array}$ \\
\hline 8,2 & 65,0 & $-10,1$ & $\begin{array}{l}10,0 \\
12,5 \\
14,5\end{array}$ & $\begin{array}{l}69,6 \\
70,9 \\
70,3\end{array}$ & $\begin{array}{l}-3,2 \\
-1,4 \\
-2,2\end{array}$ \\
\hline
\end{tabular}

Résultats de l'analyse de variance :

Source de la variation Carrémoyen Erreur Signification Carré moyen Erreur Signification

\begin{tabular}{|c|c|c|c|c|c|c|}
\hline$A=$ cultivar & 246,77 & 12,24 & $* *$ & 650,41 & 8,23 & $\star \star$ \\
\hline $\begin{array}{c}\mathrm{B}=\text { densité de population } \\
\text { d'avoine }\end{array}$ & 269,74 & 5,38 & $\star *$ & 12,56 & 8,84 & NS \\
\hline$C=$ interaction $A \times B$ & 17,35 & 5,38 & * & 8,24 & 8,84 & NS \\
\hline
\end{tabular}

NS = Effet non significatif $(P>0,05)$

$* \quad=$ Effet significatif $(0,01<P<0,05)$

** $=$ Effet hautement significatil $(P<0,01)$ 
l'époque de végétation des 2 espèces en concurrence. Il est en effet connu que l'avoine au stade plantule est particulièrement sensible à la concurrence des autres céréales et qu'elle ne manifeste un fort effet concurrentiel qu'à partir du tallage (Chancellor, 1976). Dans une culture de blé de printemps (Bastion), le seuil de nuisibilité du même cultivar d'avoine de printemps (Selma) était compris entre 16 et 28 plantes par $\mathrm{m}^{2}$ (Caussanel et Kafiz, 1986). L'absence d'effet densité en 1987 confirme ces résultats dans une culture de blé d'hiver. Lorsqu'une avoine se développe plus précocement dans un blé d'hiver, le seuil de nuisibilité est plus proche de celui qui a été déterminé dans l'essai de 1986 : environ 5 plantes par $\mathrm{m}^{2}$ (Gonzales-Ponce, 1982). De façon analogue, il a été démontré que la date de semis du blé influençait la diminution de rendement de cultivars de blé soumis à la concurrence d'une mauvaise herbe comme Setaria viridis (L.) P. Beauv. (Blackshaw et al., 1981) ou Avena sterilis L. ssp ludoviciana (Efthimiadis \& Skorda, 1983).

Comme l'interaction "cultivar $x$ densité» s'est montrée significative $(0,01<P<0,05)$ en 1986 , l'effet de la densité d'avoine d'hiver sur le rendement du blé a été étudié en fonction du cultivar. En reliant le rendement et le nombre de pieds d'avoine au tallage du blé, il est possible de calculer une équation de régression linéaire hautement significative $(P<0,01)$ pour 3 cultivars seulement (Fidel, Castan et Talent). Pour Capitole, l'équation de régression la mieux ajustée aux données de l'essai est de nature quadratique (Fig. 2). Dans chaque équation de régression de la perte de rendement en fonction de la densité adventice, l'intervalle de confiance de la moyen-

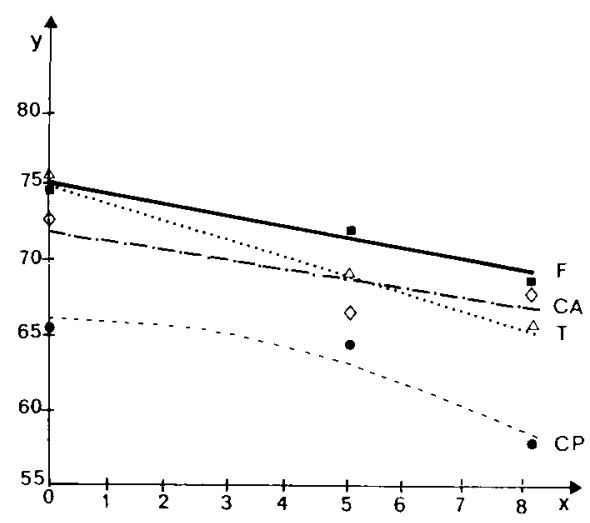

Fig. 2. Relation entre le rendement du blé ( $y$, en $q / h a)$ et le nombre de pieds d'avoine par $\mathrm{m}^{2}$ au début du tallage $(x)$ pour différents cultivars de blé (essai 1985 - 1986) (Seuls figurent les points correspondants aux moyennes de rendement pour chaque densité $D_{j}$ ).

L F = Fidel: $\widehat{y}=75,44-0,807 \times r^{2}=0,985$;

$\bullet-\ldots \mathrm{CP}=$ Capitole $: \hat{y}=66,12-0,117 x^{2} r^{2}=0,949$;

- - $-\mathrm{CA}=$ Castan : $\mathscr{y}=72,04-0,661 \times r^{2}=0,684$;

$\Delta \cdots \Delta \mathrm{T}=$ Talent $: \widehat{y}=75,38-1,242 \times r^{2}=0,998$;

$r^{2}=$ coefficient de détermination ne vraie $(P=0,05)$ a été calculé. Il est alors possible de déterminer, pour chacun des 4 cultivars, un seuil biologique de nuisibilité, exprimé ici par la densité critique d'avoine, c'est-à-dire par la densité à partir de laquelle une perte de rendement est statistiquement décelable (Caussanel, 1989). Pour Fidel, Castan et Talent, le seuil biologique de nuisibilité de l'avoine d'hiver Selma est compris entre 1 et 2 plantes par $\mathrm{m}^{2}$, mais pour Capitole il est légèrement supérieur ( 3 plantes par $\mathrm{m}^{2}$ ) dans les conditions expérimentales de l'essai 1985-1986 (Fig. 3). Des courbes de réponse très voisines ont été obtenues pour les autres variables de densité étudiées, que la densité soit la densité réelle de la parcelle ou la densité estimée sur 2 placettes, qu'elle soit mesurée au tallage ou à maturation (Mondragon, 1988).

\section{Influence de la densité d'avoine sur les com- posantes du rendement}

Dans l'essai de 1985-1986, l'augmentation de la densité d'avoine d'hiver a principalement réduit le nombre d'épis de blé par plante et le poids de 1000 grains, tous cultivars confondus (Tableau V). L'interaction "cultivar $x$ densitée n'étant significative pour aucune des composantes du rendement $(P>0,05)$, la réponse à l'ef-

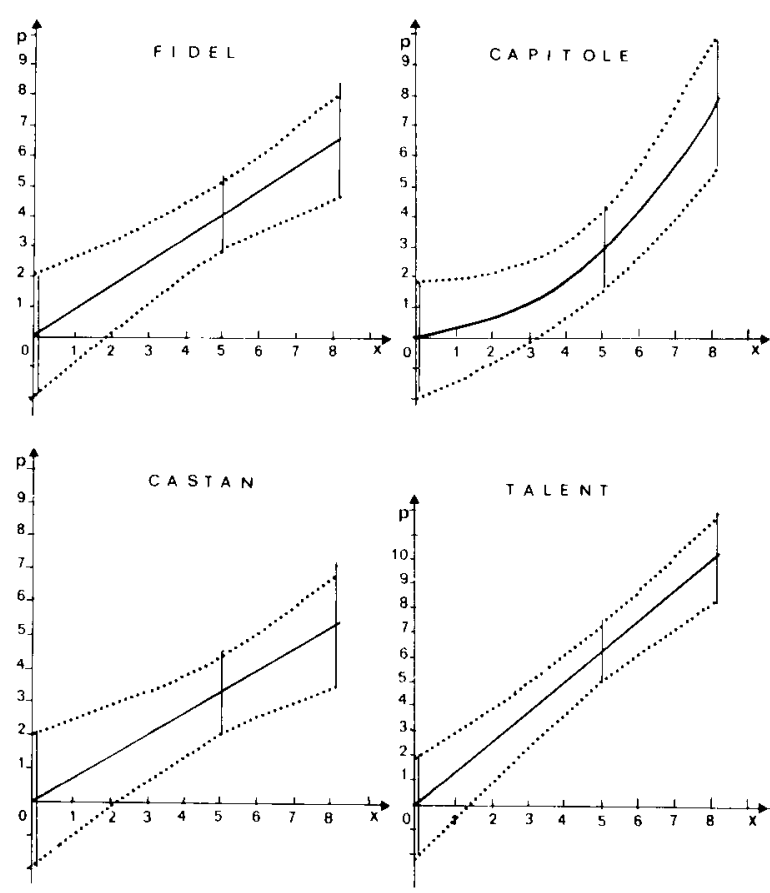

Fig. 3. Relation entre la perte de rendement du blé ( $p$, en $\mathrm{q} / \mathrm{ha}$ ) et le nombre de pieds d'avoine par $\mathrm{m}^{2}$ au début du tallage $(x)$ pour différents cultivars de blé (essai 1985-1986) Fidel : $\hat{p}=0,807 x$; Capitole : $\hat{p}=0,117 x^{2}$; Castan : $\hat{\rho}=0,661 x$; Talent : $\hat{\rho}=1,242 x$

.? Intervalle de confiance de la moyenne vraie 
Tableau V. Influence de la densité d'avoine sur les composantes du rendement et le rendement estimé de différents cultivars de blé d'hiver.

\begin{tabular}{|c|c|c|c|c|c|}
\hline $\begin{array}{l}\text { Densité d'avoine } \\
\text { par } m^{2}\end{array}$ & Fidel & Capitole & Castan & Talent & Moyenne \\
\hline
\end{tabular}

$\begin{array}{llllll}0 & 457 & 455 & 500 & 600 & 499 \\ 5,1 & 459 & 448 & 459 & 617 & 494 \\ 8,2 & 443 & 425 & 465 & 554 & 470 \\ \text { Moyenne } & 451 \mathrm{a} & 440 \mathrm{a} & 471^{\mathrm{a}} & 586 \mathrm{~b} & \end{array}$

b) Nombre d'épis de blé par plante (NEBP)

$\begin{array}{llllll}0 & 2,47 & 2,41 & 2,23 & 2,90 & 2,50 \\ 5,1 & 2,70 & 2,32 & 2,24 & 3,13 & 2,60 \\ 8,2 & 2,46 & 2,32 & 2,04 & 2,77 & 2,40 \\ \text { Moyenne } & 2,54 \mathrm{c} & 2,35 \mathrm{~b} & 2,17 \mathrm{a} & 2,93 \mathrm{~d} & \end{array}$

$\begin{array}{llllll}0 & 35,8 & 33,1 & 37,3 & 31,4 & 34,4 \\ 5,1 & 35,4 & 31,8 & 35,3 & 29,9 & 33,1 \\ 8,2 & 36,8 & 32,3 & 34,3 & 31,4 & 33,7 \\ \text { Moyenne } & 36,0^{\mathrm{b}} & 32,4 \mathrm{a} & 35,6 \mathrm{~b} & 30,9 \mathrm{a} & \end{array}$

$\begin{array}{llllll}0 & 46,3 & 47,0 & 42,0 & 39,3 & 43,7 \\ 5,1 & 45,5 & 47,3 & 41,0 & 38,2 & 43,0 \\ 8,2 & 45,2 & 46,4 & 41,4 & 37,8 & 42,7 \\ \text { Moyenne } & 45,6 \mathrm{c} & 46,9 \mathrm{c} & 41,5 \text { b } & 38,4 \text { a } & \end{array}$

\begin{tabular}{llllll}
0 & 74,8 & 70,6 & 77,6 & 72,8 & 73,9 \\
5,1 & 73,5 & 66,7 & 66,2 & 70,2 & 69,1 \\
8,2 & 73,0 & 62,1 & 65,6 & 64,8 & 66,4 \\
Moyenne & 73,7 b & 66,5 a & 69,8 ab & 69,3 ab & \\
\hline
\end{tabular}

Résultats de l'analyse de variance

Essai 1985-1986

Essai 1986-1987

\begin{tabular}{|c|c|c|c|c|c|c|c|c|c|c|}
\hline Variable & $N E B M$ & NEBP & $N G B E$ & $P M G B$ & RENE & $N E B M$ & NEBP & NGBE & $P M G B$ & RENE \\
\hline \multicolumn{11}{|l|}{ A : Cultivar } \\
\hline Carré moyen & 67569 & 1,600 & 92,20 & 215,46 & 134,87 & 466956 & 10,360 & 509,60 & 594,94 & 589,67 \\
\hline Erreur & 2294 & 0,030 & 8,60 & 2,86 & 33,93 & 2066 & 1,168 & 1,15 & 1,65 & 42,06 \\
\hline Signification & $\star *$ & $\star \star$ & $* *$ & $\star *$ & $\star$ & 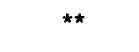 & ** & ** & ** & * \\
\hline \multicolumn{11}{|c|}{ B : Densité d'avoine } \\
\hline Carré moyen & 4833 & 0,197 & 8,89 & 4,69 & 293,46 & 1804 & 0,056 & 1,80 & 0,55 & 28,60 \\
\hline Erreur & 1958 & 0,049 & 6,80 & 1,17 & 21,03 & 1126 & 0,133 & 1,40 & 0,80 & 15,86 \\
\hline Signification & NS & * & NS & $*$ & $\star \star$ & NS & NS & NS & NS & NS \\
\hline
\end{tabular}


fet de la densité d'avoine sur le nombre d'épis de blé par plante ou sur le poids de 1000 grains est indépendante du cultivar. Aucun effet significatif $(P>0,05)$ dû à la densité d'avoine sur les composantes du rendement n'a été constaté dans l'essai de 1986-1987.

L'élaboration du rendement est physiologiquement différente pour chaque cultivar de blé d'hiver, comme le montrent les effets variétaux obtenus chaque année sur toutes les composantes $\mathrm{du}$ rendement. Le rendement estimé peut être calculé par la formule suivante :

Rendement estimé (en q/ha) $=$

$$
\text { NEBM } \times \text { NGBE } \times \text { PMGB } \times 10^{-4}
$$

où NEBM représente le nombre d'épis de blé par $\mathrm{m}^{2}$, NGBE le nombre de grains de blé par épi et PMGB le poids de 1000 grains de blé en $\mathrm{g}$, à $15 \%$ d'humidité.

Toutes densités confondues, la comparaison par le test Duncan des moyennes variétales pour chaque composante du rendement montre de nettes différences génétiques entre cultivars :

- un nombre d'épis de blé par $\mathrm{m}^{2}$ plus élevé pour Talent et plus faible pour Capitole, Fidel et Castan, ce qui confirme la différence de tallage-épi du cv Talent,

- un nombre de grains par épi plus grand pour les cultivars Fidel et Castan,

- un poids de mille grains plus important pour les cultivars Fidel et Capitole.

\section{Différences variétales de réponse à la concurrence et d'effet partenaire du blé}

Chez le blé Talent, l'évolution du développement de plantes soumises ou non à la concurrence de l'avoine montre que ni le rythme d'apparition des feuilles du brin-maître, ni celui des talles, ne présentent de différences liées à la densité d'avoine (Fig. 4a). En l'absence de concurrence avec l'avoine, le développement végétatif de ce cultivar s'est déroulé plus précocement et la capacité de tallage a été plus élevée en 1987 qu'en 1986 (Fig. 4b). En 1987, une régression du nombre de talles épiées par rapport au nombre de talles formées a été constatée pour tous les cultivars. En 1986 comme en 1987, le tallage plus important de Talent par rapport aux trois autres cultivars se traduit à la récolte par un nombre plus élevé d'épis par plante (Tableau V). Cet avantage génétique, qui a contribué à assurer une plus grande productivité du cultivar Talent, ne lui permet pas cependant de bien résister à la concurrence de l'avoine d'hiver (Fig. 3). D'autres résultats expérimentaux ont montré que des différences de pouvoir concurrentiel ne peuvent a

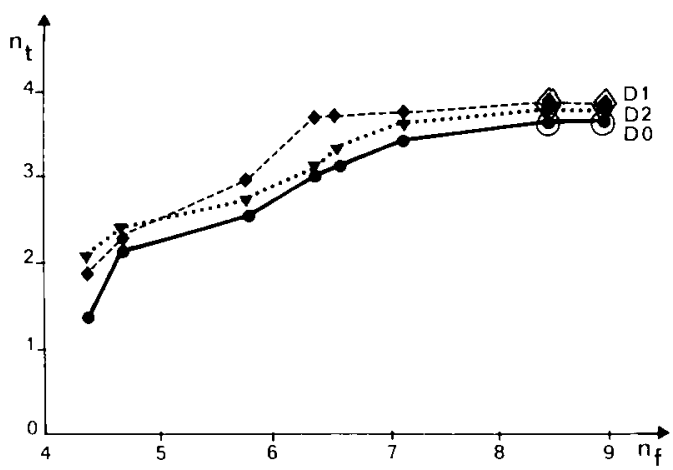

b

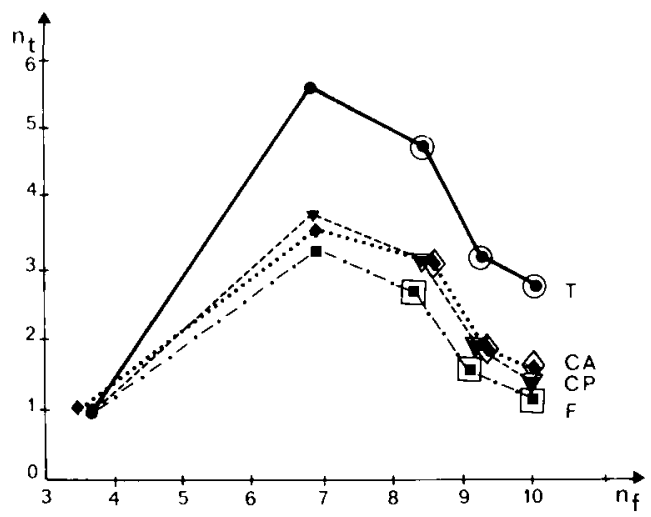

Fig. 4. Relation entre le nombre de talles $\left(n_{t}\right)$ et le nombre de feuilles $\left(n_{f}\right)$ du maître-brin par plante de blé

a) pour le cultivar Talent, à chaque densité d'avoine (essai 1985-1986) Nombre de talles : D0 ——; D1 . . . . $\mathrm{D} 2 \mathbf{\nabla} . . . \mathrm{\nabla}$. Nombre de talles épiées DO D1 $\diamond \ldots \Delta ; \mathrm{D} 2 \nabla . . . \nabla$.

b) pour les 4 cultivars de blé, à la densité Do (essai 19861987). Nombre de talles : $F=$ Fidel

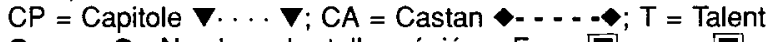

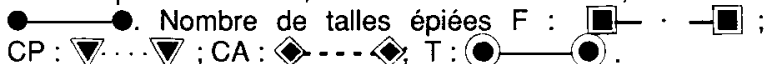

être attribuées d'une manière simple à des critères purement morphologiques, comme la hauteur de paille ou la capacité de tallage (Moss, 1985; Challaia et al., 1986).

En 1986, les analyses de biomasse des parties aériennes de blé indiquent que la biomasse de blé (de paille ou d'épis) diminue avec la densité d'avoine d'hiver alors que la biomasse totale (de blé et d'avoine) augmente (Fig. 5a). Des différences s'observent entre cultivars : si le cultivar Fidel a réagi plus tardivement à la concurrence de l'avoine, au moment du remplissage des grains, la hauteur de paille plus courte du blé Talent l'a rendu assez tôt sensible à la concurrence de l'avoine, comme le fait a été démontré chez des blés à paille très courte et à cycle biologique comparable (Wimschneider \& Bachthaler, 1979; Blackshaw et al., 1981). A ces densités faibles, l'augmentation de la densité totale de peuplement (blé et avoine) permet encore une meilleure utilisation des ressources environne- 


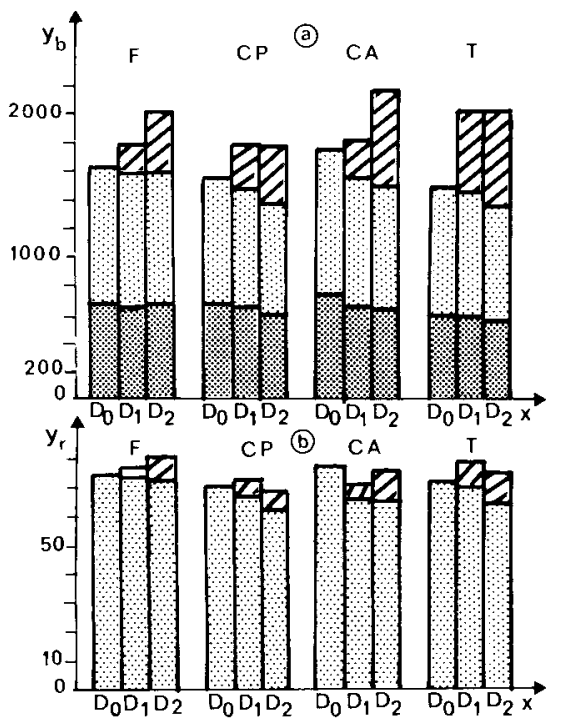

Fig. 5. Effet de la densité d'avoine d'hiver ( $x$, en nombre de pieds par $\mathrm{m}^{2}$ ) sur la production du blé et de l'avoine en concurrence en fonction du cultivar de blé (essai 1985-1986). $\mathrm{F}=$ Fidel $\mathrm{CP}=$ Capitole $\mathrm{CA}=$ Castan; $\mathrm{T}=$ Talent

a) effet sur la biomasse à maturation. $y b=$ poids de matière fraîche des parties aériennes (en $\left.\mathrm{g} / \mathrm{m}^{2}\right)$ de blé; 1 paille de blé b) effet sur le rendement.

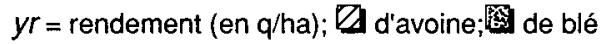

mentales, alors que pour des densités beaucoup plus élevées, la matière sèche totale des parties aériennes de céréales en concurrence varie peu (Caussanel \& Kafiz, 1986).

Les effets de densité sur le rendement estimé du blé reproduisent les effets précédents sur la biomasse à maturation du blé (Fig. 5b), mais il n'existe plus de différence significative $(P>0,05)$ liée à la densité entre les rendements totaux en grains : la concurrence entre le blé et l'avoine est devenue plus sévère pour la production de semences.

De tels résultats montrent que, lorsqu'il s'agit de comparer le pouvoir compétitif de plusieurs cultivars vis-à-vis d'une adventice, il convient d'analyser le fonctionnement intégré des 2 espèces en concurrence sur plusieurs paramètres de croissance et de développement. En effet, comme le prouvent des expériences de concurrence entre différents cultivars de blé d'hiver et Avena sterilis ssp ludoviciana (Torner et al., 1985) ou Bromus tectorum L. (Challaia et al., 1986), la perte de rendement la plus faible d'un cultivar vis-à-vis de la graminée adventice n'est pas nécessairement corrélée avec la capacité d'étouffement la plus forte de la plante cultivée envers la mauvaise herbe. La capacité d'un cultivar à supprimer la croissance des mauvaises herbes pourrait être reliée à la quantité de lumière interceptée à certains stades de développement végétatif de ce cultivar (Wicks et al., 1986).
Par ailleurs, l'augmentation de fertilisation azotée est susceptible de remplacer la suprématie d'un cultivar de blé par celle d'un autre dans leur concurrence avec Avena fatua L. (Henson \& Jordan, 1982).

\section{CONCLUSION}

Les résultats présentés montrent que le seuil de nuisibilité biologique d'une avoine d'hiver considéré comme adventice, dans une culture de blé d'hiver, varie selon l'aptitude à la concurrence du cultivar utilisé. La sensibilité variétale à la concurrence d'une avoine d'hiver (cultivar Fringante) a été examinée sous des aspects génétiques et fonctionnels pour 4 cultivars. En 1986, seul Capitole présente un rendement inférieur à celui des trois autres. Talent, qui a eu le plus fort rendement en l'absence d'avoine, a été très tôt sensible à la concurrence de celle-ci en raison de sa paille plus courte : à la récolte, le nombre d'épis par plante ou le poids de 1000 grains ont été nettement diminués. Fidel s'est montré plus résistant et la concurrence de l'avoine ne s'est exprimée que très tardivement, au moment de la formation des grains. Les pertes de rendement ont été moins élevées pour Castan dans la gamme de densités d'avoine expérimentée. Le rendement de Capitole, qui a été le plus faible en l'absence d'avoine, a subi proportionnellement moins de réduction que celui des autres cultivars en fonction de la densité d'avoine. Le seuil biologique de nuisibilité de Capitole s'est montré légèrement plus élevé que celui des autres cultivars : 3 plantes par $\mathrm{m}^{2}$ au lieu de 1 à 2 plantes par $\mathrm{m}^{2}$.

En 1987, c'est Castan qui a montré le rendement le plus faible en l'absence d'avoine, qui était ici une avoine de printemps (cultivar Selma). Les densités d'avoine de printemps, comprises entre 0 et 15 plantes par $\mathrm{m}^{2}$, n'ont pas montré d'effet sur le rendement de chacun de ces 4 cultivars, en raison de l'avantage de précocité de développement du blé sur l'avoine au moment de la levée de l'avoine de printemps. Dans les conditions de cet essai, le seuil biologique de nuisibilité de cette avoine de printemps dans le blé d'hiver a donc été supérieur à 15 plantes par $\mathrm{m}^{2}$.

Pendant les 2 années d'expérimentation, l'étude du fonctionnement intégré comparatif des plantes d'avoine et de blé a permis d'expliquer au moins partiellement les pertes de rendement constatées par les mesures des composantes du rendement et des biomasses des plantes en concurrence. De telles mesures sont essentielles pour saisir les effets dus aux variations de l'offre environnementale qui modifient chaque année le 
développement des plantes en présence, et par suite, les seuils biologiques de nuisibilité d'une adventice dominante dans une céréale.

\section{REMERCIEMENTS}

Les auteurs expriment leurs remerciements à Monsieur $M$. Bourlier pour son assistance technique, à Messieurs J.P. BLanchon, G. Durey et C. Sigwalt pour l'aide et les conseils apportés au cours de la réalisation pratique de l'essai, à Monsieur $\mathrm{A}$. Vincent pour ses conseils scientifiques dans l'étude des stades-repères du blé ainsi qu'à Monsieur G. Barralis, directeur du laboratoire de malherbologie, où ce travail a été réalisé dans le cadre d'une A.T.P. «Ecophysiologie du blé».

\section{RÉFÉRENCES}

Barralis G. (1961) Etude de la distribution des diverses espèces de folles avoines en France. Ann. Physiol. Vég. 3 (1), 39-53

Barralis G. (1977) Répartition et densité des principales mauvaises herbes en France. Document INRA SFPP. SEI - CNRA, Versailles, France, 22 pp

Blackshaw R.E., Stobbe E.H. \& Storko A.R.W. (1981) Effect of seedling dates and densities of green foxtail (Setaria viridis) on the growth and production of spring wheat (Triticum aestivum). Weed Sci. 29 (3), 212-217

Branlard G. \& Chevalet C. (1984) Sur la diversité des blés tendres cultivés en France. Agronomie, 4 (10), 933-938

Caussanel J.P. (1989) Nuisibilité et seuils de nuisibilité des mauvaises herbes dans une culture annuelle; situation de concurrence bispécifique. Agronomie 9, 219-240

Caussanel J.P. \& Kafiz B. (1986) Une méthode de détermination des seuils de nuisibilité d'une espèce adventice dans une céréale (Avena sp. $x$ blé de printemps). Proc. EWRS Symposium 1986. Economic Weed Control. pp. 83-90

Challaia H.R.E., Burnside O.C., Wicks G.A. \& Johnson V.A. (1986) Competition between winter wheat (Triticum aestivum) cultivars and downy brome (Bromus tectorum). Weed Sci. 34 (5); 689-693

Chancellor R.J. (1976) Growth and development of wild oat plants. In: Wild Oats in World Agriculture. (Price-Jones D. ed.) Agric. Res. Council, Londres, UK, pp. 89-98

Chancellor R.J. \& Peters N.C.B. (1976) Competition between wild oats and crops. In: Wild Oats in World Agriculture. (Price-Jones D. ed.) Agric. Res. Council, Londres, UK, pp. 99-112

Efthimiadis P. \& Skorda E. (1983) L'influence de la saison de l'ensemencement sur la compétition de la folle avoine avec deux variétés de blé. C.R. $12^{\circ}$ Conf. Columa. ANPP Paris, 3, 181-194
Flood R.G. \& Halloran G.M. (1984) Growth habit variation in hexaploïd wheat (Triticum aestivum) and competition with annual rye-grass (Lolium rigidum). Prot. Ecol. 6 (4), 299-305

Glauninger J. \& Holzner W. (1982) Interference between weeds and crops : a review of litterature In: Biology and Ecology of Weeds. (Holzner W. \& Numata N. eds) Publ. W. JUNK, La Haye, Pays-Bas, pp. 149-159 Gonzales-Ponce R. (1982) Competencia de Avena sterilis L. contra diversas variedades de trigo. An. Edafol. y Agrobiol. 41, (9-10), 1971-1982

Harlan J.R. (1982) Relationships between weeds and crops, In: Biology and Ecology of Weeds (Holzner W. \& Numata M. eds.) W. Junk, La Haye, Pays-Bas, pp. 91-96

Henson J.F. \& Jordan L.S. (1982) Wild oat (Avena fatua) competition with wheat (Triticum aestivum and T. turgidum durum) for nitrate. Weed Sci., 30, 297-300. Holmes H.M. \& Pfeiffer K. (1962) Some aspects of the use of barban for wild oat control in winter wheat. Weed Res. 2, 110-121

Kirby E.J.M., Appleyard D.M. \& Fellowes G. (1985) Effect of sowing date and variety on main shoot leaf emergence and number of leaves of barley and wheat. Agronomie 5 (2), 117-125

Little T.M. (1981) interpretation and presentation of results. Hort. Science 16, 637-640

Malvoisin P. (1984) Organogenèse et croissance du maître-brin du blé tendre (Triticum aestivum) du semis à la floraison. I. Relations observées entre la croissance foliaire et la différenciation des ébauches foliaires ou florales. Agronomie 4 (6), 557-564

Mondragon G. (1988) Contribution à l'étude des seuils de nuisibilité biologique de quelques espèces d'avoine dans une culture de blé. Thèse DocteurIngén., ENSAR, N. 88-2, A, 12, Univ. Rennes I, 137 pp Moss S.R. (1985) The influence of crop variety and seed rate on Alopecurus myosuröides competition in winter cereals. Proceedings 1985 Brit. Crop Protect. Conf. Weeds 2, 701-708

Snedecor G.W. \& Cochran W.G. (1971) Méthodes statistiques. lowa State University Press et ACTA, 6e édition, Paris

Torner C., Fernandez-Quintanilla C., Navarrete L. \& Sanchez M.J. (1985) Tolerancia y capacidad competitiva de diferentes variedades de trigo y cebada en presencia de Avena sterilis L. subsp. ludoviciana (Durieu. Nyeman). Informac. Tecn. Econ. Agraria 16 (59) 65-71

Wicks G.A., Ramsel R.E., Nordquist P.T., Schmidt J.N. \& Challaia H. (1986) Impact of wheat cultivars on establishment and suppression of summer annual weeds. Agron. J. 78 (1) 59-62

Wimschneider W. \& Bachthaler G. (1979) Untersuchungen über die Licktkonkurrenz zwischen Avena fatua $\mathrm{L}$. und verschiedenen Sommerweizensorten. Proc. EWRS Symp. Influence of Different Factors on the Development and Control of Weeds, Mayence, pp. 249-256 